TITLE:

\title{
Synthesis and properties of amino acid-derived optically active photo- responsive polymers
}

\section{$\operatorname{AUTHOR}(\mathrm{S}):$}

Sogawa, Hiromitsu; Terada, Kayo; Masuda, Toshio; Sanda, Fumio

\section{CITATION:}

Sogawa, Hiromitsu ... [et al]. Synthesis and properties of amino acid-derived optically active photo-responsive polymers. Polymer Bulletin 2009, 63(6): 803-813

\section{ISSUE DATE:}

2009-12

URL:

http://hdl.handle.net/2433/93014

\section{RIGHT:}

(C) 2009 Springer-Verlag.; This is not the published version. Please cite only the published version.; この論文は出版社版でありません。引用の 際には出版社版をご確認ご利用ください。 


\title{
Synthesis and Properties of Amino Acid-derived Optically Active Photo-responsive Polymers
}

\author{
Hiromitsu Sogawa, Kayo Terada, Toshio Masuda', Fumio Sanda (*) \\ Department of Polymer Chemistry, Graduate School of Engineering, Kyoto University \\ Kyoto 615-8510, Japan \\ 1 Present Address: Department of Environmental and Biological Chemistry, Faculty of \\ Engineering, Fukui University of Technology, 3-6-1 Gakuen, Fukui 910-8505, Japan \\ E-mail: sanda@adv.polym.kyoto-u.ac.jp
}

\section{Summary}

Optically active azobenzenedicarboxylic acids were synthesized from L-alanine, and the polycondensation of the diacids with $o-, m$-, $p$-xylylenediols, 5-norbornene-2,3endo,endo- and exo,exo-dimethanols, $m-, \quad p$-xylylenediamines, and trans-1,4cyclohexanediamine was carried in $N, N$-dimethylformamide. Polymers with weightaverage molecular weights of 3,300-33,700 were obtained in 63\%-quantitative yields. All the polymers reversibly isomerized the azobenzene units from trans to cis and vice versa upon UV- and visible-light irradiations. The polymers exhibited no evidence for the formation of a chiral secondary structure.

\section{Introduction}

Polymers that transform the structures by external stimuli such as temperature [1], $\mathrm{pH}$ [2], and light [3,4] gather much interest. Especially, photo-responsive polymers enable the design of highly sensitive photo-functional materials because of easy control over the irradiation wavelength, time, and intensity. Conformational changes of polymers induced by the isomerization of photochromic groups lead to the changes of physical and chemical properties including viscosity, conductivity, solubility, wettability, morphology, and so on [3-8].

Many attempts have been made to synthesize well-defined photo-responsive polymers, where azobenzene [9-12], spiropyran [13-15], and diarylethene [16-19] are commonly introduced as a photo-responsive chromophore at the main chain or side chain. For example, trans-azobenzene absorbs light around $320 \mathrm{~nm}$ based on the $\pi^{-} \pi^{*}$ transition to transform into cis-azobenzene, and the cis-isomer absorbs light based on the $\mathrm{n}-\pi^{*}$ transition around $430 \mathrm{~nm}$ to transform into trans-one reversibly, accompanying large changes of conformation and dipole moment. The cis-form also isomerizes into trans-one upon heating due to the low activation energy from cis to trans. We have previously synthesized conjugated polymers bearing azobenzene moieties in the main chain [20-23], some of which reversibly undergo cis-trans isomerization in spite of the rigid structures. We have also synthesized polyacetylenes substituted with azobenzene at the side chains [24-27]. Among them, chiral polymers form a helical structure with predominantly one-handed screw sense, and the photoisomerization of the azobenzene moieties triggers the transformation of the secondary 
structure to alternate the chiroptical properties. Amino acids are the useful biological chiral resources. Polymers containing amino acids attract attention due to their optical and biological activities [28-33]. In the course of our study on azobenzene-containing conjugated polymers, we have designed novel optically active amino acid-based polymers containing azobenzene moieties in the main chain, expecting the changes of chiroptical properties upon photo-irradiation. This article deals with the polycondensation of optically active azobenzenedicarboxylic acids with diols and diamines, and the photo-isomerization behavior of the obtained polymers (Scheme 1).

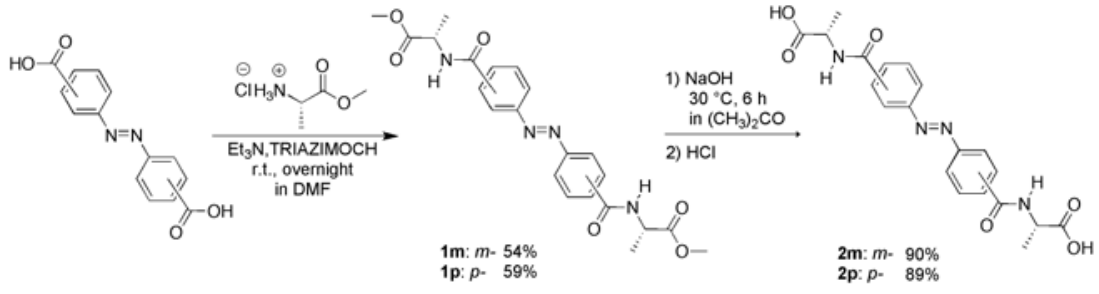

Scheme 1. Synthesis of monomers

\section{Experimental}

\section{Measurements}

${ }^{1} \mathrm{H}(400 \mathrm{MHz})$ and ${ }^{13} \mathrm{C}(100 \mathrm{MHz}) \mathrm{NMR}$ spectra were recorded on a JEOL EX-400 spectrometer. IR spectra were measured on a JASCO FT/IR-4100 spectrophotometer. Melting points (mp) were measured on a Yanaco micro melting point apparatus. Specific rotations $\left([\alpha]_{\mathrm{D}}\right)$ were measured on a JASCO DIP-100 digital polarimeter with a sodium lamp as a light source. Elemental analysis was done at the Microanalytical Center of Kyoto University. Number- and weight-average molecular weights $\left(M_{\mathrm{n}}\right.$ and $M_{\mathrm{w}}$ ) of polymers were determined by gel permeation chromatography (GPC) on TSK gel $\alpha$-3000, using a solution of $\operatorname{LiBr}(10 \mathrm{mM})$ in $N, N$-dimethylformamide (DMF) as an eluent at a flow rate of $1.0 \mathrm{~mL} / \mathrm{min}$, calibrated by polystyrene standards at $40{ }^{\circ} \mathrm{C}$. UV-vis absorption spectra were recorded on a JASCO J-820 spectropolarimeter.

\section{Materials}

All the reagents and solvents were used as purchased without purification.

\section{Synthesis of $\mathbf{1 m}$}

3,3'-Azobenzenedicarboxylic acid (1.08 g, $4.00 \mathrm{mmol}$ ) was dissolved in DMF (12 $\mathrm{mL})$, and triethylamine $(1.23 \mathrm{~mL}, 8.80 \mathrm{mmol})$ was added to the solution at room temperature. L-Alanine methyl ester hydrochloride $(1.23 \mathrm{~g}, 8.80 \mathrm{mmol})$ and 4-[4,6dimethoxy-1,3,5-triazin-2-yl]-4-methylmorpholinium chloride (TRIAZIMOCH, Tokuyama Co., $2.86 \mathrm{~g}, 8.80 \mathrm{mmol}$ ) were added to the mixture, and the resulting mixture was stirred at room temperature overnight. DMF was then distilled off using a rotary evaporator, and the residue was dissolved in $\mathrm{CH}_{2} \mathrm{Cl}_{2}$. The solution was washed with $0.5 \mathrm{M} \mathrm{HCl}$, saturated $\mathrm{NaHCO}_{3}$ aq., saturated $\mathrm{NaCl}$ aq., and then dried over anhydrous $\mathrm{MgSO}_{4}$. The residue was purified by recrystallization with methanol/ethyl 
acetate to obtain $1 \mathrm{~m}$ as orange solid in $54 \%$. Mp $192-194{ }^{\circ} \mathrm{C}$. $[\alpha]_{\mathrm{D}}+40^{\circ}(c=0.100$ $\mathrm{g} / \mathrm{dL}$ in DMF at room temperature). ${ }^{1} \mathrm{H}$ NMR (400 $\left.\mathrm{MHz} \mathrm{CDCl}_{3}\right): \delta 1.57(\mathrm{~s}, 6 \mathrm{H},-$ $\mathrm{CHCH}_{3^{-}}$), 3.82 (s, $\left.6 \mathrm{H},-\mathrm{OCH}_{3}\right), 4.80-4.87$ (m, 2H, $\left.-\mathrm{CHCH}_{3}^{-}\right), 6.83$ (d, $2 \mathrm{H},-\mathrm{CHNH}-$ ), 7.95-8.01 (m, 8H, Ar). ${ }^{13} \mathrm{C}$ NMR (100 MHz, $\left.\mathrm{CDCl}_{3}\right): \delta 18.7\left(-\mathrm{CHCH}_{3}-\right)$, $48.6(-$ $\mathrm{CHCH}_{3}-$ ), 123.2, 128.1, 136.1, 154.2 (Ar), 166.0 (-NHCO-), $173.6\left(-\mathrm{COOCH}_{3}\right)$. Anal. Calcd for $\mathrm{C}_{22} \mathrm{H}_{24} \mathrm{~N}_{4} \mathrm{O}_{6}$ : C, 59.99; H, 5.49; N, 12.72. Found: C, 59.71; H, 5.41; N, 12.76.

\section{Synthesis of $\mathbf{2 m}$}

$0.5 \mathrm{M} \mathrm{NaOH}$ aq. (8.00 mL, $4.00 \mathrm{mmol})$ was added to a suspension of $\mathbf{1 m}(0.805 \mathrm{~g}$, $1.81 \mathrm{mmol})$ in acetone $(40 \mathrm{~mL})$, and the mixture was stirred at $30{ }^{\circ} \mathrm{C}$ for $6 \mathrm{~h} .0 .5 \mathrm{M}$ $\mathrm{HCl}$ aq. (8.00 $\mathrm{mL}, 4.00 \mathrm{mmol}$ ) was added to the resultant mixture, and it was concentrated on a rotary evaporator. The residual solid was washed with ethyl acetate to obtain $2 \mathrm{~m}$ as orange solid in $86 \%$ yield. Mp $256-257^{\circ} \mathrm{C}$. $[\alpha]_{\mathrm{D}}+80^{\circ}(c=0.100 \mathrm{~g} / \mathrm{dL}$ in DMF at room temperature). ${ }^{1} \mathrm{H}$ NMR (400 MHz, DMSO- $\left.d_{6}\right): \delta 1.42$ (d, $J=7.4 \mathrm{~Hz}$, $6 \mathrm{H},-\mathrm{CHCH}_{3^{-}}$), 4.42-4.49 (m, 2H, $-\mathrm{CHCH}_{3}-$ ), 7.73 (t, $\left.J=7.8 \mathrm{~Hz}, 2 \mathrm{H}, \mathrm{Ar}\right), 8.09$ (d, $J=$ $7.6 \mathrm{~Hz}, 4 \mathrm{H}, \mathrm{Ar}$ ), 8.44 (s, 2H, Ar), 8.94 (d, $J=7.1 \mathrm{~Hz}, 2 \mathrm{H},-\mathrm{CHCH}_{3} \mathrm{NH}-$ ), 12.5 (s, 2H, $\mathrm{COOH}) .{ }^{13} \mathrm{C}$ NMR $\left(100 \mathrm{~Hz}, \mathrm{CDCl}_{3}\right): \delta 16.9\left(-\mathrm{CHCH}_{3}-\right), 48.3\left(-\mathrm{CHCH}_{3}-\right), 121.4,125.4$, 129.7, 130.6, 135.3, 151.7 (Ar), 165.3 (-NHCO-), 174.1 (-COOH). Anal. Calcd for $\mathrm{C}_{20} \mathrm{H}_{20} \mathrm{~N}_{4} \mathrm{O}_{6}$ : C, 58.25; H, 4.89; N, 13.59. Found: C, 57.96; H, 4.91; N, 13.48.

\section{Synthesis of 1p}

The title compound was synthesized from 4,4'-azobenzenedicarboxylic acid and Lalanine methyl ester hydrochloride in a manner similar to $\mathbf{1 m}$. Yield 49\%. Mp 231$233{ }^{\circ} \mathrm{C} .{ }^{1} \mathrm{H}$ NMR (400 MHz, $\mathrm{CDCl}_{3}$ ): $\delta 1.57$ (s, 6H, $-\mathrm{CHCH}_{3^{-}}$), 3.82 (s, 6H, $-\mathrm{OCH}_{3}$ ), 4.80-4.87 (m, 2H, - $\mathrm{CHCH}_{3}$ ) 6.83 (d, 2H, -CHNH-), 7.95-8.01 (m, 8H, Ar). ${ }^{13} \mathrm{C} \mathrm{NMR}$ $\left(100 \mathrm{MHz}, \mathrm{CDCl}_{3}\right): \delta 18.7\left(-\mathrm{CHCH}_{3}-\right), 48.6\left(-\mathrm{CHCH}_{3}-\right), 123.2,128.1,136.1,154.2$ (Ar), 166.0 (-NHCO-), $173.6\left(-\mathrm{COOCH}_{3}\right)$.

\section{Synthesis of $\mathbf{2 p}$}

The title compound was synthesized from $\mathbf{2 p}$ in a manner similar to $\mathbf{2} \mathbf{m}$. Yield $98 \%$. No mp was observed up to $281^{\circ} \mathrm{C}$ (decomposition). $[\alpha]_{\mathrm{D}}+120^{\circ}(c=0.100 \mathrm{~g} / \mathrm{dL}$ in DMF at room temperature). ${ }^{1} \mathrm{H}$ NMR (400 MHz, DMSO-d $\left.d_{6}\right): \delta 1.42$ (d, $J=7.7 \mathrm{~Hz}, 6 \mathrm{H}$, $-\mathrm{CHCH}_{3}-$ ), 4.39-4.48 (m, 2H, $-\mathrm{CHCH}_{3}-$ ), 8.00 (d, $J=8.3 \mathrm{~Hz}, 4 \mathrm{H}, \mathrm{Ar}$ ), 8.11 (d, $J=8.3$ $\mathrm{Hz}, 4 \mathrm{H}, \mathrm{Ar}), 8.88\left(\mathrm{~d}, J=7.1 \mathrm{~Hz}, 2 \mathrm{H},-\mathrm{CHCH}_{3} \mathrm{NH}-\right) 12.6(\mathrm{~s}, 2 \mathrm{H},-\mathrm{COOH}) .{ }^{13} \mathrm{C} \mathrm{NMR}$ $\left(100 \mathrm{MHz}, \mathrm{CDCl}_{3}\right): \delta 16.8\left(-\mathrm{CHCH}_{3}-\right), 48.3\left(-\mathrm{CHCH}_{3}-\right), 122.6,128.8,136.5,153.3$ (Ar), 165.3 (-NHCO-), 174.1 (-COOH). Anal. Calcd for $\mathrm{C}_{20} \mathrm{H}_{20} \mathrm{~N}_{4} \mathrm{O}_{6}$ : C, 58.25; $\mathrm{H}$, 4.89; N, 13.59. Found: C, 57.89; H, 4.86; N, 13.38 .

\section{Polycondensation: A typical procedure}

A solution of $2 \mathrm{~m}$ (166 mg, $0.402 \mathrm{mmol}$ ), 3 (56 mg, $0.405 \mathrm{mmol}$ ), 1-[3(dimethylamino)propyl]-3-ethylcarbodiimide hydrochloride $(\mathrm{EDC} \cdot \mathrm{HCl}, 162 \mathrm{mg}$, $0.845 \mathrm{mmol}$ ), and 4-(dimethylamino)pyridine (DMAP, $10 \mathrm{mg}, 0.081 \mathrm{mmol}$ ) in DMF $(0.8 \mathrm{~mL})$ was stirred at $30{ }^{\circ} \mathrm{C}$ for $24 \mathrm{~h}$. The resulting mixture was poured into a large amount of $0.5 \mathrm{M} \mathrm{NaHCO}_{3}$ aq. to precipitate a solid polymer. It was washed with 0.5 $\mathrm{M} \mathrm{NaHCO}_{3}$ aq., separated by filtration with a membrane filter (pore size $0.2 \mu \mathrm{m}$ ), and dried in vacuo. 
Spectroscopic Data of the Polymers

Poly(2m-3) ${ }^{1} \mathrm{H}$ NMR (400 MHz, DMSO-d f $_{6}$ : $\delta 1.44$ (br, 6H, - $\mathrm{CHCH}_{3}$ ), 4.54 (br, 2H, $-\mathrm{CHCH}_{3}-$ ), 5.20 (br, 4H, -CH $\mathrm{CH}_{2}$ ), 7.23-7.40 (br, 4H, Ar), 7.72 (br, 2H, Ar), 8.09 (br, 4H, Ar), 8.42 (br, 2H, Ar), 9.09 (br, 2H, -NHCO-). IR (cm $\left.{ }^{-1}, \mathrm{KBr}\right): 2877$ (CH), 1744 (COO), 1647 (C=O), 1528 (NHCO), 1303, 1206, 1157, 758, 691.

Poly(2m-4) ${ }^{1} \mathrm{H}$ NMR (400 MHz, DMSO-d ${ }_{6}$ ): $\delta 1.44$ (br, 6H, - $\mathrm{CHCH}_{3}$ ), 4.54 (br, 2H, $-\mathrm{CHCH}_{3}-$ ), 5.16 (br, 4H, -CH $\mathrm{CH}_{2}$ ), 7.23-7.30 (br, 4H, Ar), 7.72 (br, 2H, Ar), 8.08 (br, 4H, Ar), 8.50 (br, 2H, Ar), 9.09 (br, 2H, -NHCO-). IR (cm $\left.{ }^{-1}, \mathrm{KBr}\right): 2987$ (CH), 1745 (COO), 1653 (C=O), 1527 (NHCO), 1304, 1205, 1157, 691.

Poly(2m-5) ${ }^{1} \mathrm{H}$ NMR (400 MHz, DMSO-d $)$ ) $\delta 1.44$ (br, 6H, - $\mathrm{CHCH}_{3}$ ), 4.54 (br, 2H, $-\mathrm{CHCH}_{3}-$ ), 5.13 (br, 4H, -CH $\mathrm{CH}_{2} \mathrm{O}$ ), 7.23-7.34 (br, 4H, Ar), 7.72 (br, 2H, Ar), 7.92-8.21 (br, 4H, Ar), 8.51 (br, 2H, Ar), 9.09 (br, 2H, -NHCO-). IR ( $\left.\mathrm{cm}^{-1}, \mathrm{KBr}\right): 2986$ (CH), 1744 (COO), 1647 (C=O), 1527 (NHCO), 1306, 1209, 1157, 813.

Poly(2m-6) ${ }^{1} \mathrm{H}$ NMR (400 MHz, DMSO- $d_{6}$ ): $\delta$ 1.30-1.72 (br, 8H, - $\mathrm{CHCH}_{3}-\square$ bridge position), 2.20 (br, 2H, norbornene $\mathrm{CH}$ ), 2.82-3.35 (br, 6H, norbornene $\mathrm{CH}$, $-\mathrm{CH}_{2} \mathrm{O}-$ ), 4.46 (br, 2H, - $\mathrm{CHCH}_{3}-$ ), 6.06 (br, 2H, -CH=CH-), 7.70 (br, 2H, Ar), 8.09-8.21 (br, 4H, $\mathrm{Ar}$ ), 8.42 (br, 2H, Ar), 9.09 (br, 2H, -NHCO-). IR (cm $\left.{ }^{-1}, \mathrm{KBr}\right): 2979(\mathrm{CH}), 1746$ (COO), 1606 (C=O), 1524 (NHCO), 1302, 1210, 1156, 997.

Poly(2m-7) ${ }^{1} \mathrm{H}$ NMR (400 MHz, DMSO- $d_{6}$ ): $\delta$ 1.39-1.71 (br, 8H, $-\mathrm{CHCH}_{3}-\square$ bridge position), 2.16 (br, $2 \mathrm{H}$, norbornene $\mathrm{CH}$ ), 2.82-3.35 (br, 6H, norbornene $\mathrm{CH},-\mathrm{CH}_{2} \mathrm{O}-$ ), 4.42 (br, 2H, - $\mathrm{CHCH}_{3}$ ), 6.09 (br, 2H, -CH=CH-), 7.72 (br, 2H, Ar), 8.09-8.21 (br, 4H, Ar), 8.42 (br, 2H, Ar), 9.08 (br, 2H, -NHCO-). IR (cm $\left.{ }^{-1}, \mathrm{KBr}\right): 2980(\mathrm{CH}), 1746$ (COO), 1647 (C=O), 1536 (NHCO), 1306, 1209, 1157, 813.

Poly(2m-8) ${ }^{1} \mathrm{H}$ NMR (400 MHz, DMSO-d $)$ ): $\delta 1.41$ (br, 6H, - $\mathrm{CHCH}_{3}-$ ), 4.02 (br, 4H, $-\mathrm{CH}_{2} \mathrm{NH}-$ ), 4.55 (br, 2H, - $\mathrm{CHCH}_{3}-$ ), 7.14-7.37 (br, 4H, Ar), 7.72 (br, 2H, Ar), 8.08 (br, 4H, Ar), 8.52 (br, 2H, Ar), 8.58 (br, 2H, $-\mathrm{CH}_{2} \mathrm{NH}-$ ), 8.94 (br, 2H, -NHCO-). IR (cm ${ }^{-1}$, $\mathrm{KBr}): 2988$ (CH), 1640 (C=O), 1536 (NHCO), 1326, 1218, 692.

Poly(2m-9) ${ }^{1} \mathrm{H}$ NMR (400 MHz, DMSO-d $)$ ): $\delta 1.39$ (br, 6H, - $\mathrm{CHCH}_{3}$ ), 4.11 (br, 4H, $-\mathrm{CH}_{2} \mathrm{NH}-$ ), 5.04 (br, 2H, - $\mathrm{CHCH}_{3}-$ ), 7.14 (br, 4H, Ar), 7.71 (br, 2H, Ar), 8.11 (br, 4H, $\mathrm{Ar}), 8.46$ (br, 4H, Ar), 8.81 (br, 2H, -NHCO-), 9.93 (br, 2H, $-\mathrm{CH}_{2} \mathrm{NH}-$ ). IR ( $\mathrm{cm}^{-1}$, $\mathrm{KBr}): 2940$ (CH), 1651 (C=O), 1526 (NHCO), 1306, 758, 691.

Poly(2m-10) ${ }^{1} \mathrm{H}$ NMR (400 MHz, DMSO- $d_{6}$ ): $\delta$ 1.06-1.18 (br, 8H, $-\mathrm{C}_{6} \mathrm{H}_{8} \mathrm{H}_{2}$ ), 1.41 (br, 6H, - $\mathrm{CHCH}_{3^{-}}$), 1.72 (br, 2H, - $\mathrm{C}_{6} \mathrm{H}_{8} \mathrm{H}_{2^{-}}$), 4.45 (br, 2H, - $\mathrm{CHCH}_{3}-$ ), 7.73 (br, 2H, Ar), 8.09 (br, 4H, Ar), 8.43 (br, 4H, Ar), 8.71 (br, 2H, - $\mathrm{CH}_{2} \mathrm{NH}-$ ), 9.09 (br, 2H, -NHCO-). IR ( $\left.\mathrm{cm}^{-1}, \mathrm{KBr}\right): 2938(\mathrm{CH}), 1647$ (C=O), 1523 (NHCO), 1304, 1210, 1157.

Poly(2p-3) ${ }^{1} \mathrm{H}$ NMR (400 MHz, DMSO-d ${ }_{6}$ ): $\delta 1.44$ (br, 6H, - $\mathrm{CHCH}_{3}-$ ), 4.54 (br, 2H, $\mathrm{CHCH}_{3}-$ ), 5.17 (br, 4H, -CH $\mathrm{CH}_{2}$ ), 6.67 (br, 2H, -NHCO-), 7.22-7.41 (br, 4H, Ar), 7.94 (br, 4H, Ar), 8.09 (br, 4H, Ar). IR (cm $\left.{ }^{-1}, \mathrm{KBr}\right): 2960$ (CH), 1818 (COO), 1647 (C=O), 1604, 1515 (NHCO), 1250, 1172, 1087, 1021.

Poly(2p-4) ${ }^{1} \mathrm{H}$ NMR (400 MHz, DMSO-d ${ }_{6}$ ): $\delta 1.44$ (br, 6H, - $\mathrm{CHCH}_{3^{-}}$), 4.54 (br, 2H, $\mathrm{CHCH}_{3}-$ ), 5.17 (br, 4H, -CH $\mathrm{CH}_{2}$ ), 6.67 (br, 2H, -NHCO-), 7.22-7.41 (br, 4H, Ar), 7.94 (br, 4H, Ar), 8.09 (br, 4H, Ar). IR (cm $\left.{ }^{-1}, \mathrm{KBr}\right): 2959$ (CH), 1818 (COO), 1647 (C=O), 1604, 1516 (NHCO), 1317. 
Poly(2p-5) ${ }^{1} \mathrm{H}$ NMR (400 MHz, DMSO-d ${ }_{6}$ ): $\delta 1.45$ (br, 6H, -CHCH $3^{-}$), 4.46 (br, 2H, $\mathrm{CHCH}_{3}-$ ), 5.14 (br, 4H, -CH $\mathrm{O}_{2}$ ), 6.69 (br, 2H, -NHCO-), 7.27-7.30 (br, 4H, Ar), 8.00 (br, 4H, Ar), 8.12 (br, 4H, Ar). IR (cm-1, KBr): 2964 (CH), 1818 (COO), 1647 (C=O), 1604, 1514 (NHCO), 1317.

Poly(2p-6) ${ }^{1} \mathrm{H}$ NMR (400 MHz, DMSO-d ${ }_{6}$ ): $\delta$ 1.27-1.78 (br, 8H, - $\mathrm{CHCH}_{3}-\square$ bridge position), 2.35 (br, 2H, norbornene $\mathrm{CH}$ ), 2.71-3.35 (br, 6H, norbornene $\mathrm{CH},-\mathrm{CH}_{2} \mathrm{O}-$ ), 4.45 (br, 2H, - $\mathrm{CHCH}_{3}-$ ), 6.06 (br, 2H, -CH=CH-), 6.63 (br, 2H, -NHCO-), 8.09 (br, 4H, Ar), 8.13 (br, 4H, Ar). IR (cm $\left.{ }^{-1}, \mathrm{KBr}\right): 2984$ (CH), 1815 (COO), 1604, 1487 (NHCO), 1177, 1113.

Poly(2p-7) ${ }^{1} \mathrm{H}$ NMR (400 MHz, DMSO-d ${ }_{6}$ ): $\delta$ 1.30-1.87 (br, 8H, - $\mathrm{CHCH}_{3}-\square$ bridge position), 2.35 (br, 2H, norbornene $\mathrm{CH}$ ), 2.71-3.35 (br, 6H, norbornene $\mathrm{CH}$, - $\mathrm{CH}_{2} \mathrm{O}-$ ), 4.56 (br, 2H, - $\mathrm{CHCH}_{3}-$ ), 6.12 (br, 2H, -CH=CH-), 6.57 (br, 2H, -NHCO-), 8.08 (br, 4H, Ar), 8.12 (br, 4H, Ar). IR (cm $\left.{ }^{-1}, \mathrm{KBr}\right): 2964$ (CH), 1821 (COO), 1647 (C=O), 1604, 1509 (NHCO), 1317, 1002.

Poly(2p-8) ${ }^{1} \mathrm{H}$ NMR (400 MHz, DMSO-d $)_{6}$ ): $\delta 1.40$ (br, 6H, -CHCH $3^{-}$), 4.02 (br, 4H, $\mathrm{CH}_{2} \mathrm{NH}-$ ), 4.55 (br, 2H, - $\mathrm{CHCH}_{3}-$ ), 7.26-7.42 (br, 4H, Ar), 7.65 (br, 2H, -NHCO-), 7.99 (br, 4H, Ar), 8.10 (br, 4H, Ar), 8.82 (br, 2H, - $\mathrm{CH}_{2} \mathrm{NH}-$ ). IR ( $\left.\mathrm{cm}^{-1}, \mathrm{KBr}\right): 2940$ (CH), 1642 (C=O), 1530 (NHCO), 1297, 1159, 861.

Poly(2p-9) ${ }^{1} \mathrm{H}$ NMR (400 MHz, DMSO-d $)$ ): $\delta 1.53$ (br, 6H, - $\mathrm{CHCH}_{3}-$ ), 4.28 (br, 4H, $\mathrm{CH}_{2} \mathrm{NH}-$ ), 4.54 (br, 2H, - $\mathrm{CHCH}_{3}-$ ), 6.73 (br, 2H, -NHCO-), 7.19-7.48 (br, 4H, Ar), 8.01 (br, 4H, Ar), 8.36 (br, 4H, Ar), 8.80 (br, 2H, - $\left.\mathrm{CH}_{2} \mathrm{NH}-\right)$. IR (cm $\left.{ }^{-1}, \mathrm{KBr}\right): 2983$ (CH), 1652 (C=O), 1604, 1527 (NHCO), 1304, 1177, 1003.

Poly(2p-10) ${ }^{1} \mathrm{H}$ NMR (400 MHz, DMSO-d ${ }_{6}$ ): $\delta$ 1.06-1.18 (br, 8H, $-\mathrm{C}_{6} \mathrm{H}_{8} \mathrm{H}_{2}$ ), 1.42 (br, $6 \mathrm{H},-\mathrm{CHCH}_{3}-$ ), 1.72 (br, 2H, $-\mathrm{C}_{6} \mathrm{H}_{8} \mathrm{H}_{2}-$ ), 4.44 (br, $2 \mathrm{H},-\mathrm{CHCH}_{3}-$ ), 6.72 (br, $2 \mathrm{H},-$ NHCO-), 8.01 (br, 4H, Ar), 8.11 (br, 4H, Ar), 8.97 (br, 2H, $-\mathrm{CH}_{2} \mathrm{NH}-$ ). IR (cm ${ }^{-1}$, $\mathrm{KBr}$ ): 2939 (CH), 1653 (C=O), 1604, 1522 (NHCO), 1487, 1299, 1007.

\section{Photo-irradiation}

Photo-irradiation was carried out with a $400 \mathrm{~W}$ high-pressure mercury lamp equipped with a power source (HB-400, Fuji Glass Work) at room temperature. The appropriate wavelengths were selected either with a Pyrex glass and a UV-D33S filter (Toshiba) for irradiation at $300 \mathrm{~nm}<\lambda<400 \mathrm{~nm}$ or with an L-42 filter (Toshiba) for irradiation at $420 \mathrm{~nm}<\lambda$. Sample solutions were fed in a quartz cell, and it was placed $20 \mathrm{~cm}$ apart from the lamp. The photo-isomerization was monitored by UV-vis absorption spectroscopy.

\section{Results and discussion}

Polycondensation

The polycondensation of $\mathbf{2 m}$ and $\mathbf{2 p}$ with diols 3-7 and diamines 8-10 was carried out using EDC $\cdot \mathrm{HCl}$ as a condensation agent in the presence of DMAP in DMF at $30{ }^{\circ} \mathrm{C}$ for $24 \mathrm{~h}$ (Scheme 2). As summarized in Table 1, polymers with $M_{\mathrm{w}}$ 's of 3,300-33,700 

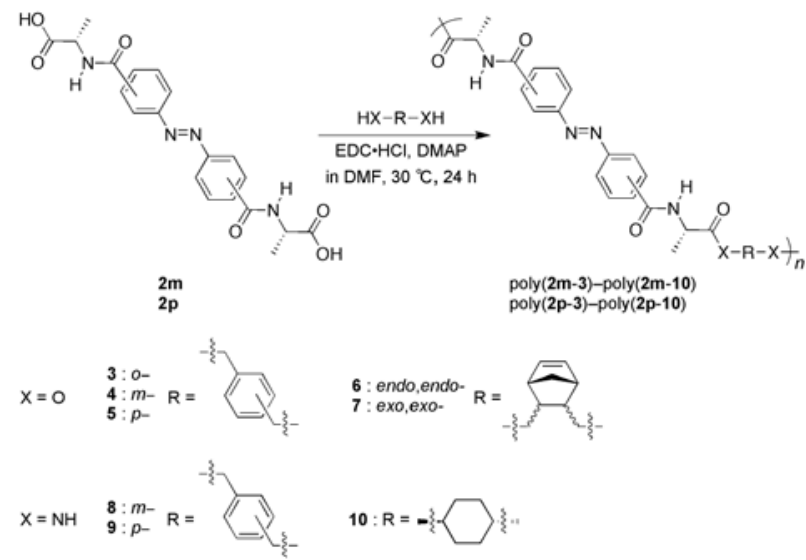

Scheme 1. Polycondensation of $\mathbf{2 m}$ and $\mathbf{2 p}$ with diols 3-7 and diamines 8-10

Table 1. Polymerization of $\mathbf{1}$ and $\mathbf{2}^{\mathrm{a}}$

\begin{tabular}{|c|c|c|c|c|}
\hline \multirow{2}{*}{ Monomer } & \multicolumn{4}{|c|}{ Polymer } \\
\hline & Yield (\%) & $M_{w}{ }^{\mathrm{d}}$ & $M_{w} / M_{n}^{\mathrm{d}}$ & {$[\alpha]_{D}{ }^{f}(\operatorname{deg})$} \\
\hline $2 m+3$ & $89^{b}$ & 4,800 & 1.8 & 10 \\
\hline $2 m+4$ & $86^{\mathrm{b}}$ & 7,500 & 1.7 & 6 \\
\hline $2 m+5$ & $86^{\mathrm{b}}$ & 8,100 & 2.2 & 0 \\
\hline $2 m+6$ & $80^{\mathrm{b}}$ & 6,600 & 1.8 & 2 \\
\hline $2 m+7$ & $70^{\mathrm{b}}$ & 4,500 & 1.9 & 3 \\
\hline $2 m+8$ & $65^{c}$ & 3,300 & 1.4 & 4 \\
\hline $2 m+9$ & $95^{\mathrm{c}}$ & 19,600 & 3.2 & 4 \\
\hline $2 m+10$ & $63^{c}$ & 7,600 & 1.5 & 8 \\
\hline $2 p+3$ & $86^{\mathrm{b}}$ & 8,000 & 2.1 & 6 \\
\hline $2 p+4$ & $99^{\mathrm{b}}$ & 20,400 & 4.5 & $-1^{\mathrm{g}}$ \\
\hline $2 p+5$ & $99^{\mathrm{b}}$ & 13,100 & 2.6 & $33^{g}$ \\
\hline $2 p+6$ & $99^{\mathrm{b}}$ & $-^{e}$ & $-^{\mathrm{e}}$ & $-^{\mathrm{e}}$ \\
\hline $2 p+7$ & $84^{\mathrm{b}}$ & 33,700 & 6.4 & $3^{g}$ \\
\hline $2 p+8$ & $75^{\mathrm{c}}$ & 3,500 & 1.5 & 55 \\
\hline $2 p+9$ & $78^{\mathrm{c}}$ & 23,800 & 3.3 & 16 \\
\hline $2 p+10$ & $85^{c}$ & 8,200 & 2.5 & 15 \\
\hline $\begin{array}{l}{ }^{\mathrm{a}} \text { Conditions: } \\
\text { at } 30{ }^{\circ} \mathrm{C} \text { for } 24 \\
\text { based on poly } \\
{ }^{\mathrm{f}} \text { Measured by } \\
{[\alpha]_{\mathrm{D}} \text { of monom }}\end{array}$ & $\begin{array}{l}l_{0}=[3-1 \\
\mathrm{O}_{3} \text { aq.-it } \\
\text { indard; } \\
\text { at room } \\
80 \text { deg, } 2\end{array}$ & $\begin{array}{l}\text { I, [EDC } \\
\text { rt. }{ }^{c} 0.5 \\
\text { r soluti } \\
\text { Ire, } c= \\
\text { g. }\end{array}$ & $\begin{array}{l}\text { M, [D } \\
\text { luble } \mathrm{p} \\
(10 \mathrm{~m} \\
\mathrm{DMF}\end{array}$ & $\begin{array}{l}0.1 \mathrm{M} \text { in } \mathrm{D} \\
\text { imated by } \\
\text { Jot determir } \\
\text { ured in } \mathrm{DM}\end{array}$ \\
\hline
\end{tabular}


were obtained in 63-99\% yields. Except for poly(2p-6), the polymers were soluble in DMF and DMSO while insoluble in hexane and toluene (Table 2). Poly(2p-6) became insoluble in DMF after isolation even though it was soluble during the polycondensation. The para-azobenzene-derived polymers tended to be less soluble than the meta-counterparts, presumably due to the larger stiffness of the para-linked backbone [34].

Table 2. Solubility of the polymers ${ }^{\mathrm{a}}$

\begin{tabular}{ccccccccc}
\hline Polymer & Hexane & Toluene & $\mathrm{CHCl}_{3}$ & $\mathrm{CH}_{2} \mathrm{Cl}_{2}$ & $\mathrm{THF}$ & $\mathrm{MeOH}$ & $\mathrm{DMF}$ & DMSO \\
\hline Poly(2m-3) & - & - & + & \pm & + & \pm & + & + \\
Poly(2m-4) & - & - & + & \pm & + & \pm & + & + \\
Poly(2m-5) & - & - & \pm & \pm & + & \pm & + & + \\
Poly(2m-6) & - & - & \pm & \pm & \pm & \pm & + & + \\
Poly(2m-7) & - & - & \pm & \pm & \pm & \pm & + & + \\
Poly(2m-8) & - & - & - & - & - & \pm & + & + \\
Poly(2m-9) & - & - & - & - & \pm & \pm & + & + \\
Poly(2m-10) & - & - & \pm & - & - & \pm & + & + \\
\hdashline Poly(2p-3) & - & - & - & - & + & - & + & + \\
Poly(2p-4) & - & - & \pm & \pm & + & \pm & + & + \\
Poly(2p-5) & - & - & \pm & \pm & \pm & \pm & + & + \\
Poly(2p-6) & - & - & - & - & - & - & - & \pm \\
Poly(2p-7) & - & - & - & - & - & - & + & + \\
Poly(2p-8) & - & - & - & - & - & \pm & + & + \\
Poly(2p-9) & - & - & - & - & - & - & + & + \\
Poly(2p-10) & - & - & - & - & - & - & + & + \\
\hline Symols: & - & - & - & - & + & + & +
\end{tabular}

${ }^{\mathrm{a}}$ Symbols: +, soluble; -, insoluble; \pm , partly insoluble.

Photo-isomerization

Polymer solutions in DMF were irradiated with UV light at a wave length of $300 \mathrm{~nm}<$ $\lambda<400 \mathrm{~nm}$ at $20^{\circ} \mathrm{C}$. The isomerization of azobenzene unit was monitored by UVvis absorption spectroscopy. As shown in Figure 1, poly(2m-3) strongly absorbed light at $320 \mathrm{~nm}$, which is attributable to the $\pi-\pi^{*}$ transition band of trans-azobenzene units. This absorption remarkably decreased the intensity upon UV-light irradiation. Simultaneously, a weak absorption attributable to the $n-\pi^{*}$ transition band of cisazobenzene units appeared around $440 \mathrm{~nm}$, and increased the intensity. Poly(2p-3) also exhibited the photo-isomerization in a manner similar to poly(2m-3) as shown in Figure 1, but the degree of isomerization was much lower. It was estimated that the trans/cis contents of azobenzene units of poly(2m-3) and poly(2p-3) were 41/59 and 83/17, respectively, after UV-light irradiation in DMF for $32 \mathrm{~min}$, based on the assumption that all azobenzene units existed in trans form at the initial state, and the 
absorption around 320-340 nm was derived from trans-azobenzene units [35]. Also the monomer $\mathbf{2 m}$ and $\mathbf{2 p}$ had 23/77 and 40/60 of trans/cis contents, respectively, after 32 min UV-light irradiation in the same conditions. It is likely that para-linked azobenzene units are unfavorable to photo-isomerization compared with meta-linked ones, and seem to be amplified by becoming the polymer. This is because para-linked azobenzene units in the trans-form are more conjugated than meta-linked ones. In fact, the $\lambda_{\max }$ and band edge of the $\pi-\pi^{*}$ transition band of poly(2p-3) appeared at $340 \mathrm{~nm}$, $20 \mathrm{~nm}$ longer than that of poly(2m-3). It presumed that this conjugation effects suppressed the photo-isomerization of para-linked polymer.
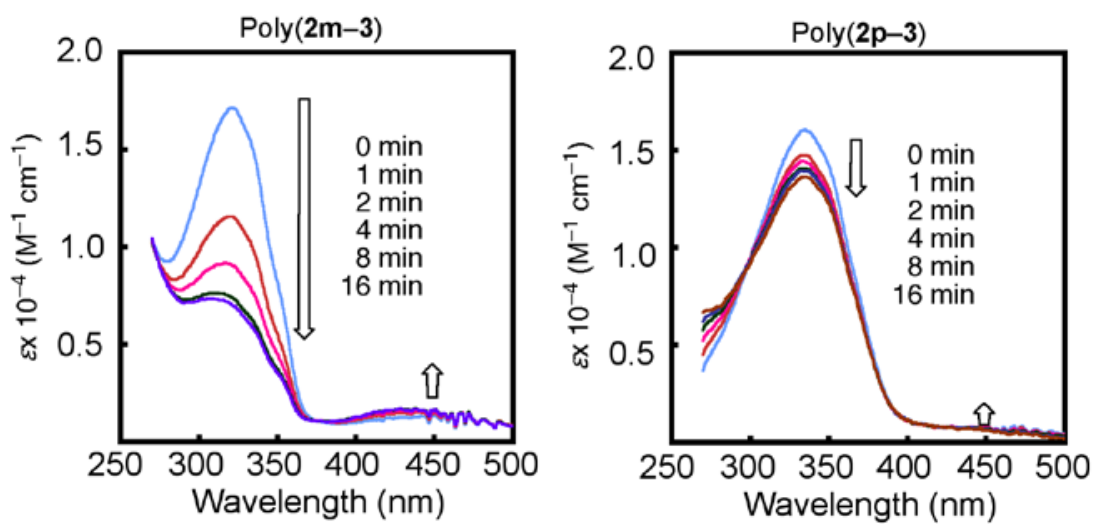

Figure 1. UV-vis spectra of poly(2m-3) and poly(2p-3) with irradiation at $300<\lambda<400 \mathrm{~nm}$ measured in DMF $(c=0.05 \mathrm{mM})$ at $20^{\circ} \mathrm{C}$.

Poly(2m-4)-poly(2m-10) exhibited UV-vis spectroscopic changes in a manner similar to poly(2m-3), and poly(2p-4)-poly(2p-10) did similarly to poly(2p-3). The absorption around $320 \mathrm{~nm}$ decreased to $12-64 \%$ of the initial state to reach a constant value by UV-light irradiation for 32-48 min. These spectral changes clearly show that trans-to-cis photo-isomerization of azobenzene moieties took place at the polymer main chain.

Next, the UV-light irradiated samples were further irradiated with visible light to check the reversibility of photo-isomerization of the azobenzene units. As shown in Figure 2, poly(2m-3) and poly(2p-3) gradually increased the absorption around 320$340 \mathrm{~nm}$ and decreased that around $440 \mathrm{~nm}$ as visible-light irradiation. After 8-32 min, the polymers recovered the UV-vis absorption at the pre-irradiation state. These spectral changes indicate the proceeding of reversible cis-to-trans photo-isomerization of azobenzene moieties in the polymers without degradation. As described above, the para-linked polymers, poly(2p-3)-poly(2p-10), isomerized the azobenzene units from trans to cis (12-40\%) less than the meta-linked counterparts, poly(2m-3)-poly(2m10) (55-65\%) upon UV-light irradiation.

The CD spectra of the polymers were measured in DMF $(c=0.05 \mathrm{mM})$ at $20{ }^{\circ} \mathrm{C}$ with UV- and visible-light irradiations to obtain information on the chiroptical property. Unfortunately however, no CD signal was observed before and after the photoirradiation, indicating that these polymers did not form a higher order structure. 
The fluorescene spectra of the monomers and polymers were also measured in the same conditions, but no emittion was observed before and after the photo-irradiation.


Figure 2. UV-vis spectra of poly(2m-3) and poly(2p-3) with irradiation at $420 \mathrm{~nm}<\lambda$, after irradiation at $300<\lambda<400 \mathrm{~nm}$ for $32 \mathrm{~min}$ measured in DMF $(c=0.05 \mathrm{mM})$ at $20^{\circ} \mathrm{C}$.

\section{Conclusion}

In this article, we have demonstrated the polycondensation of optically active azobenzenedicarboxylic acids with various diols and diamines. The polymers obtained reversibly isomerized the azobenzene units from trans to cis, and cis to trans upon UV- and visible-light irradiations, which were confirmed by UV-vis absorption spectroscopy. CD spectroscopic study revealed that the polymers did not form a higher-order structure before and after the photo-irradiation. A stiffer main chain such as phenyleneethynylene seems to be necessary to accomplish the preparation of photoresponsive polymers with a regulated secondary structure [36]. Further study on molecular design of photo-responsive optically active polymers is now under progress.

\section{Acknowledgement}

This research was partly supported by Iketani Science and Technology Foundation.

\section{References}

1. Maeda Y, Yamauchi H, Fujisawa M, Sugihara S, Ikeda I (2007) Langmuir 23:6561-6566.

2. Gil ES, Hudson SM (2004) Prog Polym Sci 29:1173-1222.

3. Seki T (2007) Bull Chem Soc Jpn 80:2084-2109.

4. Kumar GS, Neckers DC (1989) Chem Rev 89:1915-1925.

5. Lim HS, Han JT, Kwak D, Jin M, Cho K (2006) J Am Chem Soc 128:14458-14459.

6. Natansohn A, Rochon P (2002) Chem Rev 102:4139-4175.

7. Irie M (2000) Chem Rev 100:1685-1716.

8. Irie M (1990) Adv Polym Sci 94:27-67.

9. Smitha P, Asha SK, Pillai CKS (2005) J Polym Sci Part A: Polym Chem 43:4455-4468.

10. Acierno D, Amendola E, Bugatti V, Concilio S, Giorgini L, Iannelli P, Piotto SP (2004) Macromolecules 37:6418-6423.

11. Moniruzzaman M, Sabey CJ, Fernando GF (2004) Macromolecules 37:2572-2577.

12. Keum C, Ikawa T, Tsuchimori M, Watanabe O (2003) Macromolecules 36:4916-4923.

13. Wu S, Lu J, Zeng F, Chen Y, Tong Z (2007) Macromolecules 40:5060-5066. 
14. Suzuki T, Kato T, Shinozaki H (2004) Chem Commun 2036-2037.

15. Berkovic G, Krongauz V, Weiss V (2000) Chem Rev 100:1741-1753.

16. Nishi H, Kobatake S (2008) Macromolecules 41:3995-4002.

17. Kobatake S, Yamashita I (2008) Tetrahedron 64:7611-7618.

18. Kim E, Cho SY, Ahn K (2005) Mol Cryst Liq Cryst 430:135-145.

19. Kim E, Choi Y, Lee M (1999) Macromolecules 32:4855-4860.

20. Sanda F, Takihana Y, Nomura R, Masuda T (2004) Trans Mater Res Jpn 29:19-22.

21. Izumi A, Nomura R, Masuda T (2001) Macromolecules 34:4342-4347.

22. Izumi A, Teraguchi M, Nomura R, Masuda T (2000) Macromolecules 33:5347-5352.

23. Izumi A, Teraguchi M, Nomura R, Masuda T (2000) J Polym Sci Part A: Polym Chem 38:1057-1063.

24. Fujii T, Shiotsuki M, Inai Y, Sanda F, Masuda T (2007) Macromolecules 40:7079-7088.

25. Zhao H, Sanda F, Masuda T (2006) Polymer 47:2596-2602.

26. Sanda F, Teraura T, Masuda T (2004) J Polym Sci Part A: Polym Chem 42:4641-4647.

27. Nakai T, Karim SMA, Teraguchi M, Sanda F, Masuda T (2002) J Macromol Sci Pure Appl Chem A39:935-951.

28. Liu R, Shiotsuki M, Masuda T, Sanda F, Macromolecules ASAP (DOI: 10.1021/ma900910k).

29. Terada K, Masuda T, Sanda F (2009) Macromolecules 42:913-920.

30. Mallakpour S, Rafiee Z (2008) Iran Polym J 17:907-935.

31. Mallakpour S, Kolahdoozan M (2008) Polym J 40:513-519.

32. Mallakpour S, Khani M (2007) Polym Bull 59:587-596.

33. Maynard HD, Okada SY, Grubbs RH (2001) J Am Chem Soc 123:1275-1279.

34. Al-Muaikel NS (2003) Eur Polym J 39:1025-1033.

35. Moniruzzaman M, Talbot JDR, Sabey CJ, Fernando GF (2006) J Appl Polym Sci 100:1103-1112.

36. Khan A, Hecht S (2006) Chem Eur J 12:4764-4774. 